\title{
Otimização Multiobjetivo com regularização
}

\author{
Lizet Santa Cruz Calderón ${ }^{1}$ \\ IMECC/UNICAMP, Campinas, SP \\ José Mario Martínez² \\ IMECC/UNICAMP, Campinas, SP \\ Maria Aparecida Diniz Ehrhardt ${ }^{3}$ \\ IMECC/UNICAMP, Campinas, SP
}

\begin{abstract}
Resumo. Neste trabalho é introduzido um método de regularização de ordem $p$ para encontrar pontos estacionários fracos de problemas de otimização multiobjetivo com restrições. Sob condições de Hölder nas derivadas das funções objetivo, são obtidos resultados numéricos baseados nos 35 problemas de e Moré, Garbow e Hilstrom para o caso particular de $p=2$ e $p=3$
\end{abstract}

Palavras-chave. Otimização multiobjetivo, Complexidade, Hölder continuidade.

\section{Introdução}

Na otimização multiobjetivo procuramos resolver problemas de minimização de duas ou mais funções objetivo, considerando que quase nunca existe uma solução que minimize todas estas funções ao mesmo tempo, ou seja, enquanto na otimização escalar o valor ótimo é único, não podemos dizer o mesmo para problemas multiobjetivo porque uma solução que minimiza uma das funções geralmente não minimiza alguma ou todas as funções objetivo restantes (ver [6]). Algoritmos para resolver problemas de OM não lineares são iterativos. Dada uma iteração $x^{k}$, é calculado um ponto $x^{k+1}$ tal que $f_{i}\left(x^{k+1}\right)$ seja suficientemente menor que $f_{i}\left(x^{k}\right)$ para todo $i \in I$. Isso é feito definindo um modelo para cada $f_{i}(x)-f_{i}\left(x^{k}\right)$ em torno de $x^{k}$ e minimizando, aproximadamente, o máximo destes modelos (ver [3]). Fliege e Svaiter [2] parecem ser os primeiros em usar essa abordagem para resolver problemas multiobjetivo. Neste trabalho será feito uma análise do desempenho do Algoritmo proposto em [5] aplicado a testes numéricos baseados nos problemas de Moré, Garbow e Hilstrom [1], com dois valores diferentes de $p(p=2, p=3)$, onde a única condição para as restrições é que estas sejam suficientemente simples, tornando possível a obtenção da solução aproximada dos subproblemas, e gerando soluções viáveis com satisfação moderada de otimilidade.

\section{Análise de Complexidade para Problemas Multiobjetivo com Restrições}

Em [5] foi introduzido um algoritmo para problemas multiobjetivo com restrições e foi mostrado que o algoritmo gera, para o problema proposto, soluções aproximadas com precisão $\epsilon$ depois de

\footnotetext{
1lizet1910@gmail.

${ }^{2}$ martinez@ime.unicamp.br.

${ }^{3}$ cheti@ime.unicamp.br.
} 
realizar $O\left(\epsilon^{\frac{-p+\beta}{p+\beta-1}}\right)$ iterações.

Vamos considerar o seguinte problema:

$$
\begin{array}{cl}
\operatorname{minimizar} & F(x) \\
\text { sujeita a } & h_{E}(x)=0, \\
& h_{I}(x) \leq 0
\end{array}
$$

onde $F: \mathbb{R}^{n} \rightarrow \mathbb{R}^{m}, h_{E}: \mathbb{R}^{n} \rightarrow \mathbb{R}^{q}, h_{I}: \mathbb{R}^{n} \rightarrow \mathbb{R}^{s}$. Defina $F(x)=\left[f_{1}(x), \ldots, f_{m}(x)\right]$, com $f_{i}: \mathbb{R}^{n} \rightarrow \mathbb{R}$ funções cujas p-ésimas derivadas são Hölder contínuas $\forall i=1, \ldots, m$. Assumiremos que $p \in\{1,2,3, \ldots\}, L>0, \delta>0$ e $\beta \in(0,1]$, com $p+\beta>1$.

Para todo $\bar{x} \in \mathbb{R}^{n}, i \in\{1, \ldots, m\}$, definamos $M_{\bar{x}}^{i}: \mathbb{R}^{n} \rightarrow \mathbb{R}$ ( um "modelo" de $f_{i}(x)$ em torno de $\bar{x})$. Assuma que as derivadas até ordem $p$ de $M_{\bar{x}}$ existem para todo $x \in \mathbb{R}^{n}$. Dizemos que $\bar{x}$ e $x$ satisfazem as condições (2) e (4) quando

$$
\begin{gathered}
\left\|\nabla f_{i}(x)-\nabla M_{\bar{x}}^{i}(x)\right\| \leq L\|x-\bar{x}\|^{p+\beta-1}, \\
M_{\bar{x}}^{i}(\bar{x})=f_{i}(\bar{x})
\end{gathered}
$$

e

$$
f_{i}(x) \leq M_{\bar{x}}^{i}(x)+L\|x-\bar{x}\|^{p+\beta},
$$

para todo $i=1, \ldots, m$.

Se $M_{\bar{x}}(x)$ é o polinômio de Taylor de ordem $p$ em torno de $\bar{x}$ e a $p$-ésima derivada satisfaz a condição de Hölder definida para $\beta$ e $L$, as condições (2) e (4) são satisfeitas para todo $x, \bar{x} \in \mathbb{R}^{n}$. A seguir apresentamos o Algoritmo 1 voltado para o problema (1), o qual implementa um método que minimizará aproximadamente um modelo do problema original mais um termo de regularização sujeito a restrições.

Algoritmo 1. Dados: $x^{0} \in \mathbb{R}^{n}$ um ponto viável, $\alpha \in(0,1), \epsilon \in(0,1), \delta>0, \theta>0$ e $\sigma_{\min }>0$. Inicialize $k \leftarrow 0, \sigma_{0}=\sigma_{\min }$.

Passo 1: Defina $\sigma \leftarrow \sigma_{k}$.

Passo 2: Defina $\bar{x} \leftarrow x^{k}$.

Passo 3: Construa o modelo $M_{\bar{x}}(x)$ a partir de (2)-(4).

Passo 3.1: Encontre $x \in \mathbb{R}^{n}, \gamma \in \mathbb{R}^{m}, \lambda \in \mathbb{R}^{s}, \mu \in \mathbb{R}_{+}^{q}$, tais que, se $\bar{x}=x^{k}$

$$
\begin{gathered}
M_{\bar{x}}^{i}(x)+\sigma\|x-\bar{x}\|^{p+1} \leq f_{i}(\bar{x}), \text { para todo } i=1, \ldots, m \\
\gamma \geq 0, \sum_{i=1}^{m} \gamma_{i}=1
\end{gathered}
$$

$$
\left\|\sum_{i=1}^{m} \gamma_{i} \nabla\left[M_{\bar{x}}^{i}(x)+\sigma\|x-\bar{x}\|^{p+1}\right]+h_{E}^{\prime}(x)^{T} \lambda+h_{I}^{\prime}(x)^{T} \mu\right\| \leq \theta\|x-\bar{x}\|^{p},
$$

onde

$$
\begin{gathered}
\left\|\min \left\{\mu,-h_{I}(x)\right\}\right\| \leq \delta, \\
\left\|h_{E}(x)\right\| \leq \delta \text { e }\left\|h_{I}(x)_{+}\right\| \leq \delta .
\end{gathered}
$$

Passo 4: Se $\left\|\sum_{i=1}^{m} \gamma_{i} \nabla f_{i}(x)+h_{E}^{\prime}(x)^{T} \lambda+h_{I}^{\prime}(x)^{T} \mu\right\| \leq \epsilon$, pare.

Passo 5: Se a condição de decréscimo suficiente

$$
f_{i}(x) \leq f_{i}\left(x^{k}\right)-\frac{\alpha \epsilon^{\frac{p+1}{p}}}{2^{\frac{p+1}{p}}(p+2)^{\frac{p+1}{p}} \sigma^{\frac{1}{p}}}
$$


não é satisfeita para algum $i$, faça $\sigma \leftarrow 2 \sigma$, e volte para o Passo 2. Caso contrário vá para o Passo 6 .

Passo 6: Defina $x^{k+1}=x, k \leftarrow k+1, \sigma_{k}=\sigma$, e vá ao Passo 1.

Como $M_{\frac{i}{x}}^{i}(x)$ é um modelo para $f_{i}(x)$, é razoável pedir um $x$ viável tal que as componentes de $M_{\bar{x}}(x)$ diminuam tanto quanto seja possível. Isso leva a minimizar $\max \left\{M_{\bar{x}}(x)-M_{\bar{x}}(\bar{x}), \ldots, M_{\bar{x}}^{m}(x)-\right.$ $\left.M_{\bar{x}}^{m}(\bar{x})\right\}$ sujeito à viabilidade. Equivalentemente, queremos resolver, aproximadamente,

$$
\begin{aligned}
\min & z \\
\text { s.a } & M_{\bar{x}}^{i}(x)-M_{\bar{x}}^{i}(\bar{x}) \leq z, i=1, \ldots, m, \\
& h_{E}(x)=0, \\
& h_{I}(x) \leq 0 .
\end{aligned}
$$

Se as restrições (12)-(14) satisfazem uma condição de qualificação, a solução de (11)-(14) satisfaz as condições de KKT ( [4]). Mas KKT para (11)-(14) implicam as condições (6)-(9) com $\sigma=0$, $\theta=0$ e $\delta=0$. Portanto (6)-(9) satisfazem as condições de KKT aproximada para o problema

$$
\begin{aligned}
\min & \max \left\{M_{\bar{x}}^{1}(x)-M_{\bar{x}}^{1}(\bar{x}), \ldots, M_{\bar{x}}^{m}(x)-M_{\bar{x}}^{m}(\bar{x})\right\}+\sigma\|x-\bar{x}\|^{p+1} \\
\text { s.a } & h_{E}(x)=0, \\
& h_{I}(x) \leq 0 .
\end{aligned}
$$

Teorema 1. Suponha que $x^{k}$ é uma iteração calculada pelo Algoritmo 1, $\alpha \in(0,1)$ e (2)-(9) são satisfeitas para $\bar{x}=x^{k}, x, \gamma, \lambda$ e $\mu$. Então

$$
\left\|\sum_{i=1}^{m} \gamma_{i} \nabla f_{i}(x)+h_{E}^{\prime}(x)^{T} \lambda+h_{I}^{\prime}(x)^{T} \mu\right\| \leq(\theta+(p+1) \sigma)\|x-\bar{x}\|^{p}+L\|x-\bar{x}\|^{p+\beta-1} .
$$

Além disso, se

$$
\left\|\sum_{i=1}^{m} \gamma_{i} \nabla f_{i}(x)+h_{E}^{\prime}(x)^{T} \lambda+h_{I}^{\prime}(x)^{T} \mu\right\| \geq \epsilon
$$

$$
\sigma \geq \max \left\{\theta, \epsilon^{\frac{\beta-1}{p+\beta-1}} \max \left\{\frac{2^{\frac{-\beta+1}{p+\beta-1}}}{p+2} L^{\frac{p}{p+\beta-1}}, \frac{L^{\frac{p}{p+\beta-1}}}{(1-\alpha)^{\frac{p}{p+\beta-1}}(2 p+4)^{\frac{\beta-1}{p+\beta}}}\right\}\right\},
$$

tem-se

$$
\begin{gathered}
\sigma\|x-\bar{x}\|^{p} \geq \frac{\epsilon}{2 p+4}, \\
f_{i}(x) \leq f_{i}(\bar{x})-\alpha \sigma\|x-\bar{x}\|^{p+1}, \forall i=1, \ldots, m, \\
f_{i}(x) \leq f_{i}(\bar{x})-\frac{\alpha \epsilon^{\frac{p+1}{p}}}{(2 p+4)^{\frac{p+1}{p}} \sigma^{\frac{1}{p}}} .
\end{gathered}
$$

Demonstração: Ver [5].

Teorema 2. Suponha que as hipóteses do Teorema 1 são satisfeitas. Então a iteração $x^{k+1}$ está bem definida e, tomando

$$
\left.\left.c_{p}=\min \left\{\frac{1}{[2(p+2)]^{\frac{p+1}{p}}} \frac{1}{2 \theta}, \frac{1}{[2(p+2)]^{\frac{p+1}{p}}\left\{2 \operatorname { m a x } \left\{\frac{2 \frac{-\beta+1}{p+\beta-1}}{p+2} \frac{p}{p+\beta-1}\right.\right.}, \frac{L^{\frac{p}{p+\beta-1}}}{(1-\alpha)^{\frac{p}{p+\beta-1}}(2 p+4)^{\frac{\beta-1}{p+\beta-1}}}\right\}\right\}^{\frac{1}{p}}\right\},
$$


temos que, para todo $i=1, \ldots, m$,

$$
f_{i}\left(x^{k+1}\right) \leq f_{i}\left(x^{k}\right)-\alpha c_{p} \epsilon^{\frac{p+\beta}{p+\beta-1}}
$$

Demonstração: Ver [5]

Teorema 3. Suponha que as hipóteses do Teorema 1 são satisfeitas para todo $x^{k}$ calculado pelo Algoritmo 1. Seja $c_{p}$ definida como no Teorema 2. Então, dado $f_{\text {target }} \in \mathbb{R}$ arbitrário e $i \in$ $\{1, \ldots, m\}$, depois de, no máximo

$$
\left\lfloor\left(f_{i}\left(x^{0}\right)-f_{\text {target }}\right) \frac{\epsilon^{\frac{-p+\beta}{p+\beta-1}}}{\alpha c_{p}}\right\rfloor
$$

iterações, o Algoritmo 1 calcula $x \in \mathbb{R}^{n}, \lambda \in \mathbb{R}^{m}, \mu \in \mathbb{R}_{+}^{m}, \gamma \in \mathbb{R}_{+}^{m}, \sum_{i=1}^{m} \gamma_{i}=1$, que verificam $f_{i}(x) \leq f_{\text {target }}$ ou

$$
\begin{gathered}
\left\|\sum_{i=1}^{m} \gamma_{i} \nabla f_{i}(x)+h_{E}^{\prime}(x)^{T} \lambda+h_{I}^{\prime}(x)^{T} \mu\right\| \leq \epsilon, \\
\left\|h_{E}(x)\right\| \leq \delta,\left\|h_{I}(x)_{+}\right\| \leq \delta \text { e }\left\|\min \left\{\mu,-h_{I}(x)\right\}\right\| \leq \delta .
\end{gathered}
$$

Demonstração: Ver [5].

\section{Resultados Numéricos}

Com o objetivo de analisar o desempenho da abordagem proposta em [5], realizamos a implementação do Algoritmo 1 no MatLab, versão R2019a. Para resolver, em cada iteração $k$, o subproblema (12)-(14), escolhemos a rotina fmincon do MatLab, com a opção que implementa um algoritmo de ponto interior, e com os parâmetros usuais da rotina. Com base nos problemas da coleção Moré, Garbow e Hillstrom, geramos nosso conjunto de testes em que os termos $f_{1}, \cdots, f_{m}$ da nossa função objetivo $F$ são as funções que definem os problemas de quadrados mínimos não lineares descritos e introduzimos limitantes para as variáveis.

Usamos $\alpha=0.1, \epsilon=1 . e-05, \delta=0.1, \theta=100$ e $\sigma_{\min }=1$. Foram escolhidas caixas arbitrariamente, nas quais a única condição sobre elas é conter a solução dos problemas dados para o caso de quadrados mínimos. Observando o número de iterações externas e internas na tabela 1, verificamos que a versão do Algoritmo 1 com $p=3$ realiza, para cada ponto inicial escolhido, menos iterações externas e internas que a versão com $p=2$, na maioria dos casos. No entanto, esta observação é feita sem levar em conta que, ainda que tenham sido usados os mesmos pontos iniciais para todos os testes com os modelos baseados nos polinômios de Taylor de ordem 2 e de ordem 3, as soluções obtidas são, em geral, diferentes. Assim, para uma análise um pouco mais precisa, resumimos, na Tabela 2 , os casos em que ambas as versões (com $p=2$ e $p=3$ ) levam à mesma, ou aproximadamente à mesma, solução, a partir de um mesmo ponto inicial dado. As duas últimas colunas desta tabela exibem o número de iterações externas realizadas pelo algoritmo, com os diferentes polinômios de Taylor. Enquanto a versão com os polinômios de Taylor de ordem 2 realiza um total de 37 iterações externas nestes testes, a versão usando os polinômios de Taylor de ordem 3 performa um total de 22 iterações externas para os mesmos problemas, representando uma redução de aproximadamente $40 \%$ no que se refere às iterações externas, refletindo que, trabalhar com modelos de maior ordem pode tornar o algoritmo mais eficiente em termos de número de iterações. Somente omitimos os pontos iniciais e os correspondentes valores da função objetivo para o problema 19, pois este problema possui 11 variáveis e 65 termos em $F$. Para os 3 pontos iniciais escolhidos o algoritmo parou pelo critério de estacionariedade. 
Tabela 1: Resultados do Algoritmo 1 para polinômio de Taylor de ordem 2 e de ordem 3.

\begin{tabular}{|c|c|c|c|c|c|}
\hline & & $\mathrm{T} 3$ & & $\mathrm{~T} 2$ & \\
\hline $\mathrm{NP}$ & $x^{0}$ & Outer & Inner & Outer & Inner \\
\hline \multirow[t]{3}{*}{1} & $x_{I}^{0}=(1,-2)$ & 2 & 28 & 4 & 38 \\
\hline & $x_{I I}^{0}=(-1.5,-2)$ & 1 & 10 & 2 & 22 \\
\hline & $x_{I I I}^{0}=(-2,-0.8)$ & 1 & 11 & 1 & 13 \\
\hline \multirow[t]{3}{*}{2} & $x_{I}^{0}=(-2,2)$ & 11 & 48 & 14 & 57 \\
\hline & $x_{I I}^{0}=(-2,1)$ & 8 & 37 & 11 & 40 \\
\hline & $x_{I I I}^{0}=(-2,2.5)$ & 1 & 14 & 2 & 22 \\
\hline \multirow[t]{3}{*}{3} & $x_{I}^{0}=(0,1)$ & 3 & 48 & 15 & 376 \\
\hline & $x_{I I}^{0}=(1,0)$ & 15 & 207 & 16 & 161 \\
\hline & $x_{I I I}^{0}=(-1,0.5)$ & 1 & 10 & 2 & 16 \\
\hline \multirow[t]{3}{*}{4} & $x_{I}^{0}=(-1,-1)$ & 1 & 8 & 1 & 9 \\
\hline & $x_{I I}^{0}=(0,-1)$ & 1 & 5 & 1 & 9 \\
\hline & $x_{I I I}^{0}=(1,0.5)$ & 1 & 24 & 2 & 26 \\
\hline \multirow[t]{3}{*}{5} & $x_{I}^{0}=(-3,-3)$ & 1 & 1 & 4 & 22 \\
\hline & $x_{I I}^{0}=(-3,-2)$ & 3 & 6 & 5 & 23 \\
\hline & $x_{I I I}^{0}=(3,-2.5)$ & 1 & 1 & 1 & 5 \\
\hline \multirow[t]{3}{*}{6} & $x_{I}^{0}=(5,5)$ & 1 & 2 & 1 & 3 \\
\hline & $x_{I I}^{0}=(5,4)$ & 6 & 21 & 8 & 28 \\
\hline & $x_{I I I}^{0}=(5,-5)$ & 1 & 1 & 7 & 14 \\
\hline \multirow[t]{3}{*}{7} & $x_{I}^{0}=(-3,2,-1)$ & 4 & 23 & 48 & 34 \\
\hline & $x_{I I}^{0}=(-2.5,0,-3)$ & 1 & 9 & 2 & 17 \\
\hline & $x_{I I I}^{0}=(-1.5,2,-3)$ & 1 & 19 & 3 & 20 \\
\hline \multirow[t]{3}{*}{8} & $x_{I}^{0}=(-1,0,1)$ & 1 & 7 & 5 & 54 \\
\hline & $x_{I I}^{0}=(-2,-1,0)$ & 16 & 144 & 18 & 126 \\
\hline & $x_{I I I}^{0}=(1,-1,3)$ & 9 & 99 & 11 & 103 \\
\hline \multirow[t]{3}{*}{9} & $x_{I}^{0}=(-2,2,1)$ & 1 & 4 & 3 & 35 \\
\hline & $x_{I I}^{0}=(-1,0,3)$ & 6 & 41 & 7 & 24 \\
\hline & $x_{I I I}^{0}=(-1,2,-0.5)$ & 4 & 27 & 8 & 42 \\
\hline \multirow[t]{3}{*}{10} & $x_{I}^{0}=(-4,1,-3)$ & 1 & 5 & 2 & 13 \\
\hline & $x_{I I}^{0}=(1,1,-1)$ & 1 & 11 & 3 & 19 \\
\hline & $x_{I I I}^{0}=(-4,0,-1)$ & 1 & 12 & 1 & 14 \\
\hline \multirow[t]{3}{*}{11} & $x_{I}^{0}=(3,0.5,4)$ & 1 & 3 & 2 & 7 \\
\hline & $x_{I I}^{0}=(-4,2,0)$ & 1 & 4 & 2 & 9 \\
\hline & $x_{I I I}^{0}=(4,-4,0)$ & 1 & 3 & 2 & 11 \\
\hline \multirow[t]{3}{*}{12} & $x_{I}^{0}=(-2,3,4)$ & 2 & 15 & 4 & 23 \\
\hline & $x_{I I}^{0}=(0,-3,0.5)$ & 1 & 5 & 3 & 17 \\
\hline & $x_{I I I}^{0}=(-3,2,4)$ & 4 & 14 & 11 & 14 \\
\hline \multirow[t]{3}{*}{13} & $x_{I}^{0}=(-3,-1,1,1)$ & 9 & 126 & 10 & 130 \\
\hline & $x_{I I}^{0}=(-2,2,0,1)$ & 6 & 90 & 9 & 126 \\
\hline & $x_{I I I}^{0}=(-1,-3,2,-4)$ & 1 & 9 & 2 & 21 \\
\hline \multirow[t]{3}{*}{14} & $x_{I}^{0}=(-1,-3,2,0)$ & 2 & 19 & 3 & 44 \\
\hline & $x_{I I}^{0}=(-2,0,-1,1)$ & 3 & 22 & 11 & 209 \\
\hline & $x_{I I I}^{0}=(1,-2,0,3)$ & 2 & 30 & 5 & 55 \\
\hline \multirow[t]{3}{*}{15} & $x_{I}^{0}=(-4,-2,-1,1)$ & 2 & 17 & 5 & 80 \\
\hline & $x_{I I}^{0}=(-5,1,0,-3)$ & 1 & 9 & 4 & 41 \\
\hline & $x_{I I I}^{0}=(-2,0,-4,-1)$ & 4 & 64 & 7 & 91 \\
\hline \multirow[t]{3}{*}{16} & $x_{I}^{0}=(-1,3,0,1)$ & 1 & 11 & 3 & 42 \\
\hline & $x_{I I}^{0}=(-2,0,1,2)$ & 3 & 33 & 5 & 60 \\
\hline & $x_{I I I}^{0}=(-3,-1,-1,-2)$ & 4 & 36 & 7 & 28 \\
\hline \multirow[t]{3}{*}{17} & $x_{I}^{0}=(0,-1,2,-2,-4)$ & 1 & 16 & 3 & 57 \\
\hline & $x_{I I}^{0}=(-3,2,1,0,3)$ & 1 & 38 & 2 & 44 \\
\hline & $x_{I I I}^{0}=(2,-1,-4,0,4)$ & 1 & 17 & 2 & 19 \\
\hline
\end{tabular}




\begin{tabular}{|c|c|c|c|c|c|}
\hline & & T3 & & $\mathrm{T} 2$ & \\
\hline $\mathrm{NP}$ & $x^{0}$ & Outer & Inner & Outer & Inner \\
\hline \multirow[t]{3}{*}{18} & $x_{I}^{0}=(-2,-3,-1,0,0,1)$ & 1 & 23 & 5 & 105 \\
\hline & $x_{I I}^{0}=(1,-1,0,0,-2,-1)$ & 1 & 13 & 2 & 21 \\
\hline & $x_{I I I}^{0}=(-3,1,0,0,-2,-1)$ & 1 & 18 & 2 & 21 \\
\hline \multirow[t]{3}{*}{19} & $x_{1}^{0}$ & 1 & 7 & 5 & 13 \\
\hline & $x_{I I}^{0}$ & 1 & 2 & 3 & 5 \\
\hline & $x_{I I I}^{0}$ & 2 & 5 & 3 & 9 \\
\hline \multirow[t]{3}{*}{20} & $x_{I}^{0}=(-2,3,-2.5,0.5)$ & 1 & 15 & 2 & 21 \\
\hline & $x_{I I}^{0}=(-3.5,1,-1,2.5)$ & 1 & 12 & 4 & 54 \\
\hline & $x_{I I I}^{0}=(3,-4,0,1)$ & 1 & 10 & 2 & 15 \\
\hline \multirow[t]{3}{*}{21} & $x_{I}^{0}=(-3,2,-1,0)$ & 1 & 14 & 3 & 22 \\
\hline & $x_{I I}^{0}=(1,4,-2,-4)$ & 1 & 18 & 5 & 43 \\
\hline & $x_{I I I}^{0}=(-3,-2,4,0)$ & 1 & 5 & 2 & 15 \\
\hline \multirow[t]{3}{*}{22} & $x_{I}^{0}=(1,0,-2,-4)$ & 4 & 64 & 3 & 32 \\
\hline & $x_{I I}^{0}=(-2,-3,1,1)$ & 6 & 84 & 3 & 31 \\
\hline & $x_{I I I}^{0}=(-4,1,0,0)$ & 3 & 9 & 7 & 53 \\
\hline \multirow[t]{3}{*}{23} & $x_{I}^{0}=(0,-1,2,0)$ & 3 & 34 & 6 & 65 \\
\hline & $x_{I I}^{0}=(1,-2,0,-0.5)$ & 1 & 11 & 4 & 63 \\
\hline & $x_{I I I}^{0}=(0.5,-1.5,2,0)$ & 2 & 31 & 3 & 39 \\
\hline \multirow[t]{3}{*}{24} & $x_{I}^{0}=(-3,0,1,0)$ & 7 & 99 & 7 & 99 \\
\hline & $x_{I I}^{0}=(2,-1,-2.5,0)$ & 2 & 26 & 5 & 81 \\
\hline & $x_{I I I}^{0}=(-1,2,0,-3)$ & 5 & 49 & 6 & 58 \\
\hline \multirow[t]{3}{*}{25} & $x_{I}^{0}=(4,2.5,-1,3)$ & 2 & 12 & 6 & 20 \\
\hline & $x_{I I}^{0}=(-2,0,0,1)$ & 3 & 19 & 4 & 37 \\
\hline & $x_{I I I}^{0}=(5,1.5,3.5,-1)$ & 1 & 10 & 3 & 36 \\
\hline \multirow[t]{3}{*}{26} & $x_{I}^{0}=(2.5,-3,-1,0)$ & 1 & 5 & 4 & 60 \\
\hline & $x_{I I}^{0}=(-2,3,-1.5,-3)$ & 2 & 22 & 3 & 34 \\
\hline & $x_{I I I}^{0}=(0,1,-2,-1.5)$ & 5 & 29 & 8 & 70 \\
\hline \multirow[t]{3}{*}{27} & $x_{I}^{0}=(-1,0,-3,2,4)$ & 1 & 13 & 2 & 18 \\
\hline & $x_{I I}^{0}=(5,-2,1,2.5,3)$ & 1 & 11 & 3 & 24 \\
\hline & $x_{I I I}^{0}=(3,-3,4,-1.5,0)$ & 1 & 13 & 2 & 21 \\
\hline \multirow[t]{3}{*}{28} & $x_{I}^{0}=(-2,0,2,-1)$ & 1 & 6 & 5 & 34 \\
\hline & $x_{I I}^{0}=(0,0.5,-2,-1.5)$ & 5 & 63 & 6 & 61 \\
\hline & $x_{I I I}^{0}=(1,2,-0.5,1.5)$ & 4 & 48 & 7 & 79 \\
\hline \multirow[t]{3}{*}{29} & $x_{I}^{0}=(-3,0,5,-2.5,1)$ & 7 & 35 & 10 & 59 \\
\hline & $x_{I I}^{0}=(-2,5,-3,3,1)$ & 2 & 25 & 4 & 37 \\
\hline & $x_{I I I}^{0}=(4,2.5,-1.5,3,-3)$ & 3 & 38 & 4 & 49 \\
\hline \multirow[t]{3}{*}{30} & $x_{I}^{0}=(0,2.5,-1.5,1)$ & 2 & 15 & 3 & 19 \\
\hline & $x_{I I}^{0}=(-2,1.5,-1,3)$ & 1 & 9 & 6 & 41 \\
\hline & $x_{I I I}^{0}=(2,-0.5,-1,0)$ & 1 & 7 & 2 & 13 \\
\hline \multirow[t]{3}{*}{31} & $x_{I}^{0}=(-2,0,2,1.5)$ & 1 & 3 & 5 & 54 \\
\hline & $x_{I I}^{0}=(-2,2.5,-1.5,3)$ & 1 & 11 & 3 & 39 \\
\hline & $x_{I I I}^{0}=(-1,2,0,-0.5)$ & 3 & 47 & 6 & 138 \\
\hline \multirow[t]{3}{*}{32} & $x_{I}^{0}=(-1,0,2,4)$ & 3 & 42 & 6 & 55 \\
\hline & $x_{I I}^{0}=(1,1.5,-1.5,-2)$ & 1 & 16 & 3 & 51 \\
\hline & $x_{I I I}^{0}=(3,-0.5,4,-2)$ & 2 & 8 & 4 & 32 \\
\hline \multirow[t]{3}{*}{33} & $x_{I}^{0}=(0,-1,2,-3)$ & 1 & 8 & 2 & 19 \\
\hline & $x_{I I}^{0}=(2,-2,0,-1.5)$ & 2 & 17 & 3 & 27 \\
\hline & $x_{I I I}^{0}=(-2,-1.5,0,-3)$ & 1 & 6 & 1 & 8 \\
\hline \multirow[t]{3}{*}{34} & $x_{I}^{0}=(-3,-2.5,2.5,1.5)$ & 1 & 6 & 2 & 8 \\
\hline & $x_{I I}^{0}=(4,1,-0.5,0)$ & 1 & 9 & 2 & 12 \\
\hline & $x_{I I I}^{0}=(2,1,3,-3.5)$ & 1 & 8 & 2 & 10 \\
\hline \multirow[t]{3}{*}{35} & $x_{I}^{0}=(-4.5,0,-3)$ & 4 & 63 & 7 & 147 \\
\hline & $x_{I I}^{0}=(1,-1,-2)$ & 7 & 77 & 11 & 110 \\
\hline & $x_{I I I}^{0}=(-3,-2,1)$ & 5 & 85 & 6 & 94 \\
\hline
\end{tabular}


Tabela 2: Resultados do Algoritmo 1 para polinômios de ordem 2 e de ordem 3

\begin{tabular}{lccc}
\hline NP & $x^{0}$ & Taylor 2 & Taylor 3 \\
\hline 2 & $x_{I}^{0}=(-2,2)$ & 14 & 11 \\
\hline 10 & $x_{I}^{0}=(-4,1,-3)$ & 2 & 1 \\
& $x_{I I}^{0}=(1,1,-1)$ & 3 & 1 \\
& $x_{I I I}^{0}=(-4,0,-1)$ & 1 & 1 \\
\hline 11 & $x_{I}^{0}=(3,0.5,4)$ & 2 & 1 \\
\hline 20 & $x_{I I I}^{0}=(3,-4,0,1)$ & 2 & 1 \\
\hline 23 & $x_{I}^{0}=(0,-1,2,0)$ & 6 & 3 \\
& $x_{I I}^{0}=(1,-2,0,-0.5)$ & 4 & 1 \\
& $x_{I I I}^{0}=(0.5,-1.5,2,0)$ & 3 & 2 \\
\hline
\end{tabular}

\section{Conclusões}

Neste trabalho apresentamos a aplicabilidade do Algoritmo 1, realizando sua implementação computacional em MatLab. O objetivo principal desta implementação foi testar os modelos que usam derivadas terceiras da função objetivo, ou seja, o caso em que $p=3$, e derivadas segundas, ou seja, o caso em que $p=2$. Os resultados dos experimentos indicaram que a versão do Algoritmo $1 \operatorname{com} p=3$ se mostra mais eficiente do que aquela com $p=2$. Porém, devemos destacar que o uso de modelos de ordem $p=3$ (ou de ordem superior) torna as iterações mais caras. Seria necessário o registro do tempo de execução gasto pelo algoritmo para uma análise de eficiência mais precisa.Além disso, note que, diferentes escolhas de parâmetros produzem diferentes soluções eficientes e, dada uma escolha particular, a solução correspondente vem da resolução de um problema de otimização escalar padrão. Em trabalhos futuros, espera-se ampliar a complexidade para problemas de otimização não padrão , como equilíbrio, dois níveis e otimização multinível.

\section{Referências}

[1] J. J. Moré, and B.S. Garbow, and K. E. Hillstrom, Testing unconstrained optimization software, ACM Transactions on Mathematical Software, 7: 17-41, 1981.

[2] J. Fliege, and B. F. Svaiter. Steepest descent methods for multicriteria optimization, Math. Methods Oper., 2000. DOI: 10.1007/s001860000043.

[3] J. M. Martínez. On high-order model regularization for constrained optimization, SIAM J. optim, 2017. DOI: 10.1137/17M1115472.

[4] J. M. Martínez, and S. A. Santos. Métodos computacionais de otimização, $20^{\circ}$ Colóquio Brasileiro de Matemática, 1995.

[5] L. Calderón, M. A. Diniz-Ehrhardt, and J. M. Martínez. On high-order model regularization for multiobjective optimization, Optimization Methods and Software, 2020. DOI: 10.1080/10556788.2020.1719408.

[6] Y. Sawaragi, and H. Nakayama, and T. Tanino. Theory of Multiobjective Optimization. Orlando, San Diego, New York, London, 1985. 